\title{
Collective narratives catalyse cooperation
}

\author{
Chaitanya S. Gokhale ${ }^{1}$, Joseph Bulbulia ${ }^{2,3}$, Marcus Frean ${ }^{4}$ \\ ${ }^{1}$ Research Group for Theoretical Models of Eco-evolutionary Dynamics \\ Department of Evolutionary Theory, \\ Max-Planck Institute for Evolutionary Biology, 24306 Plön, Germany, \\ ${ }^{2}$ School of Psychology, Faculty of Science, Victoria University of Wellington \\ ${ }^{3}$ Max Planck Institute for the Science of Human History, Jena, Germany \\ ${ }^{4}$ School of Engineering and Computer Science, \\ Victoria University of Wellington, New Zealand
}

\begin{abstract}
Humans invest in fantastic stories - mythologies. Recent evolutionary theories suggest that cultural selection may favour moralising stories that motivate prosocial behaviours. A key challenge is to explain the emergence of mythologies that lack explicit moral exemplars or directives. Here, we resolve this puzzle with an evolutionary model in which arbitrary mythologies transform a collection of egoistic individuals into a cooperative. Importantly, in finite populations, reflecting relative to contemporary population sizes of hunter-gatherers, the model is robust to the cognitive costs in adopting fictions. This approach resolves a fundamental problem across the human sciences by explaining the evolution of otherwise puzzling amoral, nonsensical, and fictional narratives as exquisitely functional coordination devices.
\end{abstract}

15 Keywords: social evolution | culture | narratives | beliefs | stag-hunt 


\section{Introduction}

Human culture is a bewildering array of complex yet intuitively consistent, mutually 8 beneficial arrangements. From reproductive divisions of labour and alloparenting to collective foraging, information-sharing, education, and defence, humans are champions of cooperation. Large-scale cooperation traces to a deep ancestral past, built on the back of highly social hunter-gatherer modes of existence [1, 2, 3]. It has long been argued that moralising mythologies were a central feature of our cooperative adaptive complex. According to the supernatural punishment hypothesis [4, 5], natural and cultural selection targeted beliefs in the reality of supernatural policing, leading to a remarkable diversity of religious belief systems. According to these scholars, the prevalence of religious beliefs is explained by religion's cooperative effects. Specifically, beliefs in supernatural enforcers promote obedience to cooperative norms [6, 7]. A key challenge, then, is to explain the cultural persistence of a-moral, morally ambiguous, and anti-social mythologies.

For example, in Māori culture, the primordial deities Rangi and Papa are connected in a loving embrace that encompasses all of reality. The world is created when one of their children, Tane, pushes his parents apart, thus giving rise to the separation of the sky (Rangi) and earth (Papa). Since then the primordial couple has lived in longing for each other with little or no regard for their children or, for that matter, mortals. It is difficult to derive any clear moral from this story. Indeed, many cultural mythologies exhibit morally problematic features. Consider another example, in Aztec mythology, the feathered serpent god Quetzalcoatl is described as having set himself on fire the day after an intoxicating evening in which he performed sexual acts on his sister Quetzalpetlatl. Both the god's incest and the self-immolation are arguably morally excessive for a deity. Incest avoidance does not appear to be challenging to motivate. What, then, explains the emergence and propagation of mythologies that lack explicit moral instructions? Might such mythologies promote sociality some other way?

Previous theories speculate that amoral mythologies propagate from non-functional 
cognitive preferences for the uncanny [8]. Here, we offer a mathematical model of a process whereby even arbitrary fictions support sociality. Our model is distinctive because it demonstrates how evolutionary dynamics could favour fiction not as an accident but for its functions.

Cooperation threatened by risk: the Stag Hunt. To illustrate a fundamental evolutionary problem of cooperation, we begin, aptly, with a mythological story called "The stag hunt", which clarifies how the problem of cooperation arise even when cooperation is everyone's interest $[9,10,11,12]$. We imagine a group of hunters deciding whether to hunt hares or stags. Hunting a hare is possible alone, but results in a small payoff. Stag hunting yields a more substantial payoff but - because it requires collective action - it is threatened by risk-avoidance. In an all-or-none scenario, if even one hunter seeks to avoid the risk that cooperation fails, all hunters will fail. This reasoning makes the default equilibrium of ubiquitous hare-hunting stable because propensities toward stag hunting face downward selective pressure. How can populations transit to cooperation (stag-hunting) under these circumstances?

We might look to the Theory of Mind, or the capacity to represent the mental states of other people, for the solution. The consensus view is that the Theory of Mind was a critical step in achieving cooperation at scale [13]. In a stag hunt, I can represent that it is in your interests to coordinate, and you can do the same. However, it has long been observed that Theory of Mind may compromise a coordination problem with risk. Merely knowing that risk-avoidance threatens the success of a stag-hunt might inspire risk-avoidance. For example, suppose individuals would choose Stag with a probability of $p=0.9$. Knowing this, agents with shared intentionality can reason that the chance of $n$ choosing to hunt is $p^{n} \ll p$ and, being risk-averse, down-grade their own $p$ accordingly. Theory of Mind allows partners to represent each other's uncertainty. The capacity to represent uncertainty in the setting of risk threatens coordination, even when all parties understand that coordination is in everyone's interests $[14,15,16]$. Example of such coordination failures abound. During a pandemic, we understand that there is ample toilet paper for all. Nevertheless, we rush to the market, and a wrestling match ensues because we predict, correctly, that the pandemic 
will cue hoarding. Were we unable to represent the minds of others, no such problem would arise. Even successful collectives are fragile, and humans seem in need of reusable mechanisms to traverse from a world of risk aversion to one of cooperative alignment.

Distinct from the Theory of Mind - the capacity to represent the mental states of others - we define "inter-subjectivity" as the capacity of multiple individuals to share the same subjective beliefs [17]. Inter-subjective reality then is a belief, whether accurate or not, accepted by all parties concerned. To solve a coordination problem that involves risk, such as a stag hunt, requires an alignment of motivations. We next introduce a model in which arbitrary fictions cause a form of inter-subjectivity that supports risky cooperation.

\section{Model \& Results}

\subsection{Traversing the dilemma}

A tribe of individuals decide to form a group to go get food. The group consists of $G$ individuals, each of whom could opt to pursue a hare or the stag. If enough people are hunting stag, then the hunt will be successful and yield $\Pi_{S}$ for each active participant. A failed hunt results in no payoff at all. All hare hunters have a fixed payoff of $\Pi_{H}$ regardless of the group composition. The hare hunters payoff is, therefore, "risk-free", being achievable regardless of the decisions of the other hunters.

We label objective and alternate realities as 1 and 2 respectively, and each individual has a preference (belief) in favour of one or the other. Groups form, come to a consensus regarding reality 1 vs 2 , and each member then adopts the behaviour $(\mathrm{H}$ or S) they prefer in that consensus reality. Thus we have eight types of individual to consider, as shown in Fig. 1. We begin in a world where everyone favours objective reality and is a hare hunter, hence a population consisting of type $(\mathrm{H}, \mathrm{H}, 1)$.

Before setting off on a hunt, the group $G$ arrives at a consensus reality. A simple way to do this is to choose one member of the group and coalesce around their 


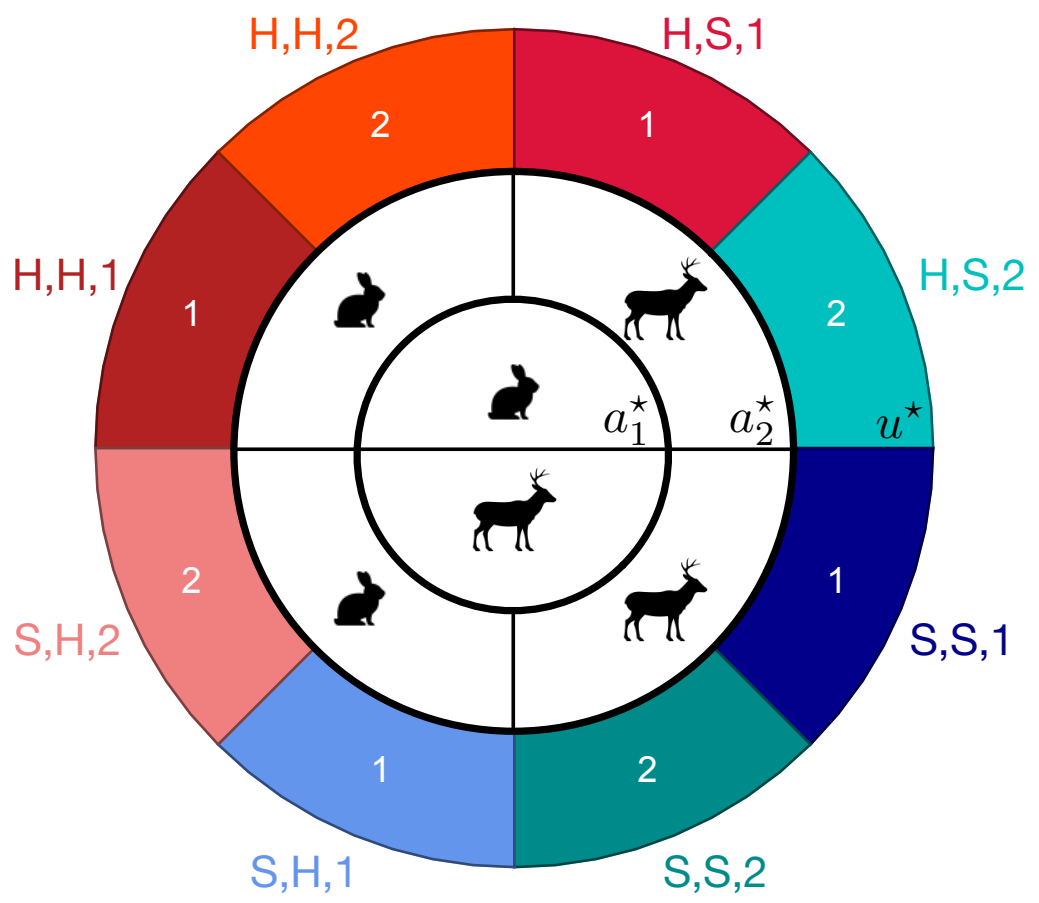

Figure 1: The types of individuals in the world. Individuals are aware of the realities 1 the objective or 2 alternate. However they believe in only one of them. The actions of the individuals in each reality could be $H$ or $S$. Thus a focal individual $(\star)$ is defined by $\left(a_{1}^{\star}, a_{2}^{\star}, u^{\star}\right)$ where $a_{i}^{\star}$ is the hunting strategy of the focal individual when in reality $i$ and $u^{\star}$ is the reality that the individual believes in. Following their own narratives, the red-tinged strategies would hunt hares, and blue-tinged, stags. 
view - a dynamics of Groupthink [18]. Humans frequently depend on the perceptions of others to inform themselves [19], and the notion of Groupthink captures the phenomenon in which a group minimises conflict by accepting a particular viewpoint without a critical evaluation of the decision or the alternatives. In this view, understanding is a contagion [20] that allows for fast decision making without conflicts. Again we illustrate using a pessimistic approach, taking a random individual as the source of the contagion. This amounts to a pure frequency-dependent ruling to reach a group decision. Group formation and composition itself can be a complex process but as this is not our focus we assume it takes place with no assortment, via random draws from the population. Some other ways of reaching a consensus are discussed in the Supplementary Material. Charismatic leaders could impose one view of reality [21], or objectivity might prevail, or everyone could simply decide for themselves. A simple majority vote is another appealing possibility supporting normative behaviour $[22,23]$. The evolutionary dynamics of conformist behaviour can very well feed into the decision making process [24].

Upon consensus, the individuals decide on their actions conditioned on that reality, and the consequences of the ensuing hunt unfold in the evolutionary dynamics.

\subsection{Evolutionary dynamics in infinite populations}

In infinitely large populations, deterministic dynamics reign supreme. Tracking all the possible types of individuals, the dynamics of the strategy evolution proceeds in a seven-dimensional simplex whose vertices correspond to strategies, with intermediate points being a mixture of different strategies.

This simplex is projected in Fig. 2 as a two-dimensional graphic. The edges connect pairs of pure strategies, and the arrows denote the direction of selection when only those strategies are in play. Neutral dynamics exist between strategies that choose the same reality and the same strategy, such as $(H, H, 2)$ and $(S, H, 2)$, or even if they have different realities but the same strategy in both, such as $(H, H, 1)$ and $(\mathrm{H}, \mathrm{H}, 2)$. Only two strategies $((\mathrm{S}, \mathrm{H}, 1)$ and $(\mathrm{H}, \mathrm{S}, 2))$ are dominant and selected over 


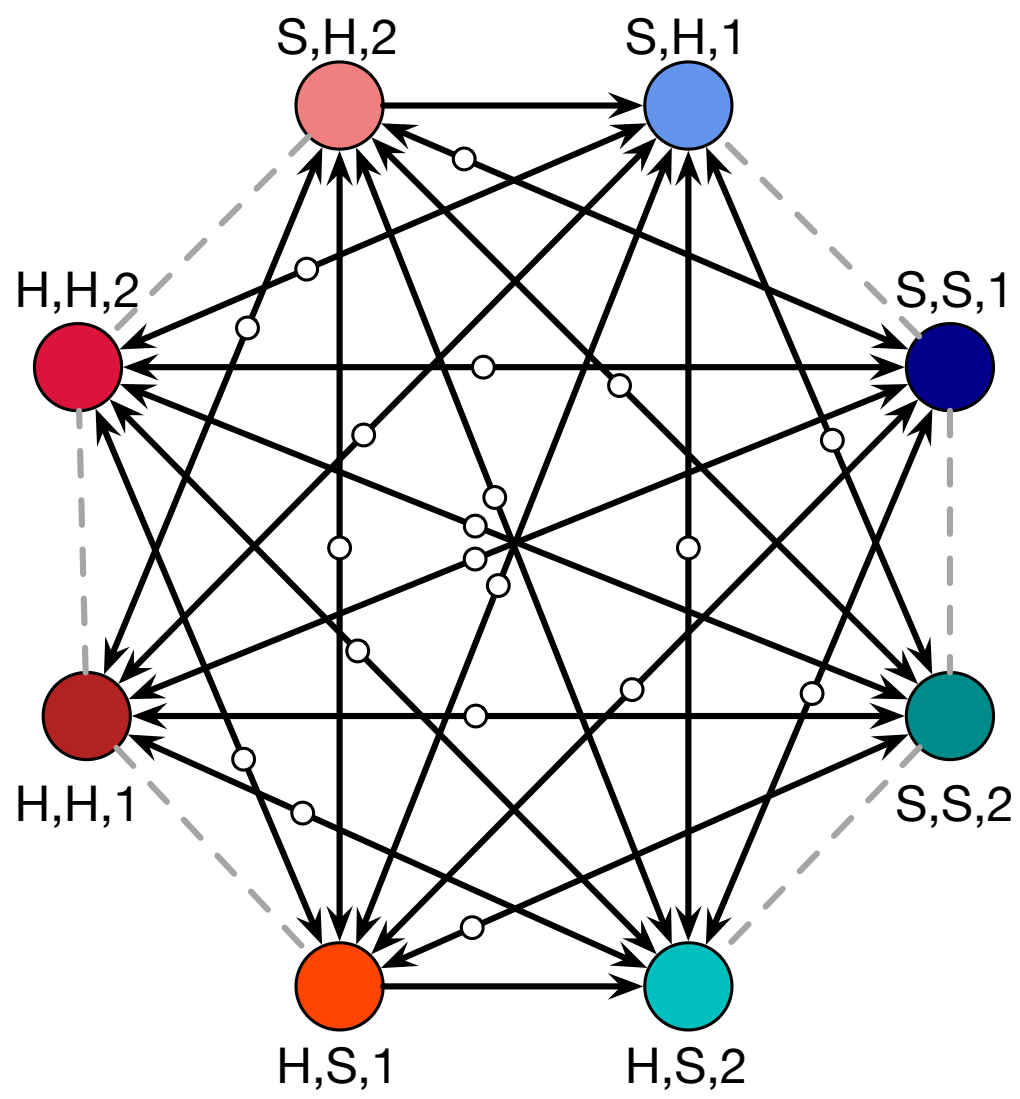

Figure 2: Dynamics on the edges of the simplex. The dashed edges are neutrally stable whereas the directed edges show dominance. The edges with an open circle on them are all bi-stable (the circle marking the position of an unstable threshold as per Fig. SI.1), hence along those edges there would be no movement once the population is at a vertex. Once the population reaches the $(\mathrm{H}, \mathrm{S}, 2)$ or $(\mathrm{S}, \mathrm{H}, 1)$ state, it gets locked into the stag equilibrium. Parameters are $G=5, M=4, P_{S}=4$ and $P_{H}=1$. 
the corresponding Hare strategies $((\mathrm{S}, \mathrm{H}, 2)$ and $(\mathrm{H}, \mathrm{S}, 1))$. The population can thus move into the Stag state where it can again evolve neutrally. The logic behind the two traversals beginning at $(\mathrm{H}, \mathrm{H}, 1)$ is as follows: (i) The bottom path allows for the neutral evolution of individuals that might hunt stags in reality 2 . This choice is not selected against because the consensus reality is always the objective one, 1 , in which they hunt hares, and thus we have neutral drift $(\mathrm{H}, \mathrm{H}, 1) \rightleftarrows(\mathrm{H}, \mathrm{S}, 1)$. From $(\mathrm{H}, \mathrm{S}, 1)$ to $(\mathrm{H}, \mathrm{S}, 2)$ inter-subjectivity can break out en masse. If the group chooses reality 2 then the payoff is larger as they will get the stag and the belief in alternate reality 2 increases until it becomes the inter-subjective reality - an accepted belief. (ii) The top path is possible in a similar fashion where it is belief in the objective reality 1 that forms the stag-inducing inter-subjective reality.

Following either path, we see that the population can go through a step in which the inter-subjectivity evolves (widespread belief in either the alternate reality (bottom) or the objective (top)) and catalyses a transition in behaviour. The dual transition is also evident in the future coexistence of the diversity of stag hunters, both believing in different realities but still trusting each other to hunt the stag. Thus while essential for mediating the transition, eventually the exact choice of belief becomes immaterial.

The mechanism leading to the bypassing of the unstable equilibrium of a stag hunt game is similar to the scenario of the evolution of cooperation by tag-based selection [25]. The narratives can be interpreted as different tags which the individuals bear and then can choose to discriminate between partners - cooperators and defectors in a Prisoner's Dilemma. However, in our case, individuals do not discriminate based on the narratives they believe. The disentangling of actions and narratives in our case leads to extremely complex dynamics, given the complicated nature of the payoff structure (see Supplementary Information (SI)). The complexity prevents us from analysing the interior of the seven-dimensional simplex in detail. However, progress is possible using a more realistic picture of the model for finite populations, together with analytical tools and computer simulations. 


\subsection{Evolutionary dynamics in finite populations}

Typical prehistoric communities were of small size. To analyse the dynamics of traits in tribes living together in countable population sizes needs different mathematics [26]. Assuming the population size of the tribe is $N$, a sample of $G$ individuals from it decide to hunt. This sampling follows a hypergeometric distribution [27, 28]. Given the population composition, the calculation of payoffs is denoted in the Appendix. As before, we assume the initial population, and hence also the hunting group $G$ consisting of $(\mathrm{H}, \mathrm{H}, 1)$ types. New strategies can arise due to mutations. From $(\mathrm{H}, \mathrm{H}, 1)$ if mutations to types such as $(\mathrm{H}, \mathrm{H}, 2)$, and $(\mathrm{H}, \mathrm{S}, 1)$ occur, they will be hunting Hare in the objective reality. Thus the fitness of these types are the same in the objective reality and thus neutral evolution proceeds.

In finite populations, drift allows for the strategies such as $(H, S, 1)$ and $(H, S, 2)$ to rise to appreciable frequencies in the population (as in [25]). However, now that $(H, S, 2)$ increases in frequency. In case the alternate belief is chosen as the group reality, the individuals who have $S$ action as their second reality option will hunt a stag. If there are more than the critical number $M$ of stag hunter in the group $G$, then the stag hunt will be successful. Given these multiple conditionals, the calculation of fitness becomes a complicated matter of bookkeeping. From the payoff of a strategy $i, \pi_{i}$, we can calculate the fitness of the strategy as $\psi_{i}=1+\omega \pi_{i}$ where $\omega$ is the intensity of selection. The selection intensity dictates the impact of the game on the fitness of a strategy. For $\omega=0$, all strategies are equal with fitness of 1 . As the value of $\omega$ increases, the game has an increasing impact on fitness. In a finite population, the Moran process translates game success into an increase in the frequency in the population (Appendix). For smaller population sizes, the stochastic effects are pronounced (see SI). Given a population size, we can explore the effect of the different selection intensities on the evolutionary dynamics as well as the eventual abundances of the strategies as illustrated in Fig 3.

Note that in the eventual distribution, mixtures of individuals believing in both realities are possible, indeed likely. Initially, lone strategists believing in the alternate 
Transient dynamics

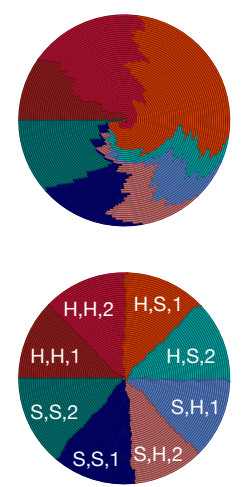

0.0
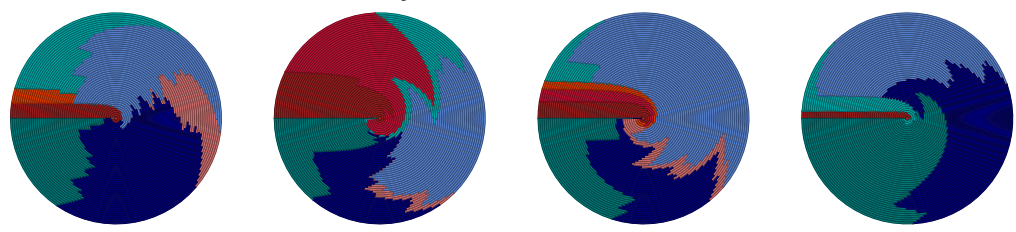

Full dynamics
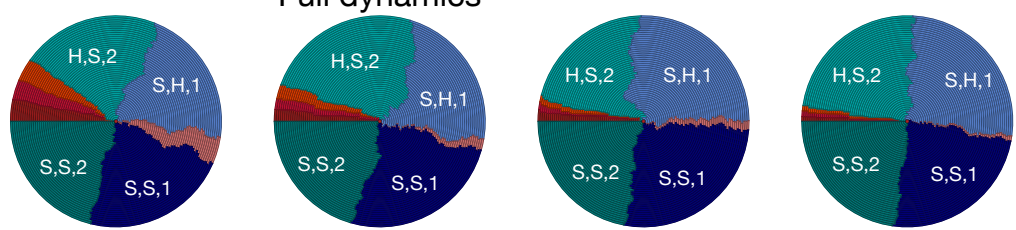

0.8

Figure 3: Abundance plots for the eight strategies in a finite population. All simulations begin with a population consisting of $(\mathrm{H}, \mathrm{H}, 1)$. Transient: For different selection intensities the initial conditions are all the same - starting in a $(\mathrm{H}, \mathrm{H}, 1)$ population. With a mutation rate of $\mu=10^{-3}$ new strategies appear and spread in the population. Every $20^{\text {th }}$ time-step up to 1000 time-steps are plotted, from inside to outside. Full: Over time $\left(5 \times 10^{6}\right.$ time-steps) the population reaches stationarity. Every $1000^{\text {th }}$ time-step is plotted. The final distribution of strategies (outermost layer of the circles) is collated in the bar-chart. Bottom: The final distribution of the strategies at time-step $5 \times 10^{6}$ is plotted as a stacked bar chart for the different section intensities. When selection intensity $\omega=0$ the dynamics is neutral and all the strategies evolve to similar abundances. When selection intensity is increased even slightly $\omega=0.2$ the population is made up predominantly of stag hunters. Note that it is not necessary that all stag hunter share the same inter-subjective reality. Parameters are $N=32, G=5, M=4, P_{S}=4$ and $P_{H}=1$. 
reality would be considered as irrational. However, since selection acts only via the action taken, the belief can spread neutrally as long as the action performed in the prevalent reality remains hare hunting. Thus, although shared narratives facilitate the transition from hare hunters to stag hunters, the narratives themselves do not necessarily fix in the population but are merely a catalyst. Even non-believers in the narrative end up hunting stags, because since the majority of the population is in a stag hunter, to hunt stags is a rational choice.

\section{Discussion \& Conclusion}

Belief in shared narratives can enforce rules on normative behaviour leading to a productive society [29]. By affecting human morality [30], beliefs can result in reduced cheating [31] and increased pro-social behaviour [32, 6, 33, 34]. We propose that since establishing a social contract became possible via the capacity for generating fictions, the potential for subjective reasoning could come under biological and cultural evolution [35, 3, 36, 37, 38, 39]. Numerous verbal or computational models exist which extol the role of prosocial mythologies in the spread of cooperation [30, 31]. Our model clarifies how mythologies that lack moralising features might nevertheless propagate as cooperative assurance mechanisms.

As with any model, ours has its limitations. We have modelled the evolution of a capacity for inter-subjective belief, assuming this capacity to be a single trait. Complex modern human behaviour is a combination of multiple traits, a package [40, 7], the contents of which can interact in to amplify or depress cooperative outcomes. Linking cultural traits resulting in a cumulative cultural evolution and exploring the evolution of this joint 'package' has been recently explored using agent-based simulations [41, 42]. Additionally, we do not explain the origin of tendencies nor attempt to clarify debates about origins of fictional capacities [43, 44, 45]. The arguments in the directions of origins need to be augmented further by studies in behavioural sciences and cultural anthropology. Combining multiple qualities, once humans acquired the ability to fictionalise their experiences, this ability would also need to be heritable. Our 
theory assumes the actions and the ability for subjective inference to be genetically encoded. Using gene-culture coevolution models, it might be possible to introduce a transmission of not only the strategies but the fictions themselves [34]. The advent of storytelling as a cultural trait perhaps could be coupled together with the passage of genes for actions. The cultural evolution of successful stories (which couple with actions that provide a fitness advantage) could lead to a thriving society [46]. Imagining stories or the ability to invent fictions indeed comes with a cognitive cost. Also, the ability to distinguish one narrative from another involves a cost as well. Hence a cost of inventing and recognising narratives is paid by all individuals - the alternate reality believers and the objective reality believers who can discern the difference. In the SI, we have explored the case where only the alternate reality believers pay the cognitive cost. A pronounced catalyst nature of an alternate reality appears in the presence of costs. After enabling a transition to the stag equilibrium in finite populations, the belief in an alternate reality disappears, leaving the population at the social optimum.

While we have not focused on the content of stories per se, the narratives of fictions in hunter-gatherer communities often focus on pro-social qualities [7, 47]. Anthropological and cognitive sciences have long highlighted the importance of belief systems in the social evolution of humans. The exact distinction between the moralising effect of mythologies versus their effects is still debated [48]. Large-scale cooperation via belief in gods, money, contracts, banks has resulted in immense growth in arts and trade, ultimately benefiting humanity. Since we show that the content of the belief does not necessarily matter, post-truths, conspiracies, and ideologies can spread as well. Beliefs might, therefore, be viewed as selfish elements. The effect on the population as a whole is inconsequential; thus, superficially, the rise and fall of beliefs might be likened to fashion dynamics [49].

Inter-subjective realities rule our world today. Differing from fashions; religions, states, nations, and companies exert enough power in the natural world to change its evolutionary course. Narrative beliefs about marital rituals, vaccination programmes, financial/government entities and money itself control the biological output of large swathes of populations around the globe $[50,51,52,53]$. The importance of narra- 
tive in economics and politics is becoming increasingly relevant [54]. Such shared narratives between many individuals, help us achieve cooperation on a large-scale. Extending our theory to include ecological dynamics would thus be a natural extension $[55,32,34]$. Populations that reach the stag equilibrium can increase their carrying capacity and competition between populations would then be important [56]. However as evidenced from the agricultural revolution, an increase in population size also comes with problems, such as lower nutritional content [57], widespread diseases $[58,59,60]$ and the further cognitive pressures for social engineering. Thus the benefit and costs of larger population size would need to be balanced out [61, 62]. If the costs are too high, an evolutionary model might revert us to the hare state, a reduction in population size and to a hunter-gatherer community.

We have chosen the stag hunt as a primary example of the problem of establishing a social contract. The model establishes a tension between a secure private gain and an unreliable but more considerable social gain. Dynamically speaking, if a model affords an unstable equilibrium between these two actions, then it will be an appropriate candidate for posing as an appropriate problem for a social contract. We have considered only two different types of realities. Once the population has surmounted one social dilemma, it will reach a plateau where a co-existence between inter-subjective beliefs is possible. Then the process can start again with a new challenge, thus leading to higher social gains but at the same time, accumulating several narratives, from personal beliefs to institutionalised systems. From hunter-gatherers to the stock-exchange, the amount of cooperation that we see in human communities is impressive in scale and achievements. By providing a mathematical model resolving a social contract, we show that such massive advent of cooperation can emerge when people have shared narratives.

\section{Acknowledgements}

We thank Charlotte Brand and Jorge Peña for suggestions on improving the paper. 


\section{References}

[1] Bocquet-Appel, J.-P. \& Bar-Yosef, O. The Neolithic demographic transition and its consequences (Springer Science \& Business Media, 2008).

[2] Bocquet-Appel, J.-P. When the World's Population Took Off: The Springboard of the Neolithic Demographic Transition. Science 333 (2011).

[3] Mcbrearty, S. \& Brooks, A. S. The revolution that wasn't: a new interpretation of the origin of modern human behavior. Journal of human evolution $39,453-563$ (2000).

[4] Swanson, G. E. The birth of the gods: The origin of primitive beliefs, vol. 93 (University of Michigan Press, 1960).

[5] Johnson, D. God is watching you: How the fear of God makes us human (Oxford University Press, USA, 2016).

[6] Shariff, A. F. \& Norenzayan, A. God Is Watching You . Psychological Science 18, 803-809 (2016).

[7] Norenzayan, A. et al. The cultural evolution of prosocial religions. Behavioral and brain sciences 39 (2016).

[8] Boyer, P. The naturalness of religious ideas: A cognitive theory of religion (Univ of California Press, 1994).

[9] Rousseau, J.-J. A Discourse on Inequality (1755).

[10] Binmore, K. G. Playing fair: game theory and the social contract (MIT Press, Cambridge, 1994).

[11] Skyrms, B. The Stag-Hunt Game and the Evolution of Social Structure (Cambridge University Press, Cambridge, 2003).

[12] Calcott, B. The other cooperation problem: Generating benefit. Biology \& Philosophy 23, 179-203 (2008). 
[13] Tomasello, M. \& Carpenter, M. Shared intentionality. Developmental science 10, 121-125 (2007).

[14] Rubinstein, A. The electronic mail game: Strategic behavior under" almost common knowledge". The American Economic Review 385-391 (1989).

[15] Binmore, K. \& Samuelson, L. Coordinated action in the electronic mail game. Games and Economic Behavior 35, 6-30 (2001).

[16] Binmore, K. Do conventions need to be common knowledge? Topoi 27, 17 (2008).

[17] Scheff, T. J. Goffman Unbound! (The Sociological Imagination). The Sociological Imagination (Routledge, 2006).

[18] Bond, M. The Power of Others: Peer Pressure, Groupthink, and How the People Around Us Shape Everything We Do (Oneworld Publications, 2015).

[19] Landauer, T. K. How much do people remember? some estimates of the quantity of learned information in long-term memory. Cognitive Science 10, 477-493 (1986).

[20] Sloman, S. \& Fernbach, P. The Knowledge Illusion: Why We Never Think Alone (Riverhead Books, 2017).

[21] Bulbulia, J. \& Frean, M. The Evolution of Charismatic Cultures. Method \& Theory in the Study of Religion 22, 254-271 (2010).

[22] Ehrlich, P. R. \& Levin, S. A. The evolution of norms. PLoS One 3, 943-948 (2005).

[23] Chalub, F. A. C. C., Santos, F. C. \& Pacheco, J. M. The evolution of norms. Journal of Theoretical Biology 241, 233-240 (2006).

[24] Denton, K. K., Ram, Y., Liberman, U. \& Feldman, M. W. Cultural evolution of conformity and anticonformity. Proceedings of the National Academy of Sciences 117, 13603-13614 (2020). 
[25] García, J., van Veelen, M. \& Traulsen, A. Evil green beards: Tag recognition can also be used to withhold cooperation in structured populations. Journal of Theoretical Biology 360, 181-186 (2014).

[26] Nowak, M. A., Sasaki, A., Taylor, C. \& Fudenberg, D. Emergence of cooperation and evolutionary stability in finite populations. Nature 428, 646-650 (2004).

[27] Gokhale, C. S. \& Traulsen, A. Evolutionary games in the multiverse. Proceedings of the National Academy of Sciences USA 107, 5500-5504 (2010).

[28] Wu, B., Traulsen, A. \& Gokhale, C. S. Dynamic properties of evolutionary multiplayer games in finite populations. Games 4, 182-199 (2013).

[29] Peoples HC, M. F. Subsistence and the evolution of religion. Human Nature 23, 253-269 (2012).

[30] Roes, F. L. \& Raymond, M. Belief in moralizing gods. Evolution and Human Behavior 24, 126-135 (2003).

[31] Shariff, A. F. \& Norenzayan, A. Mean Gods Make Good People: Different Views of God Predict Cheating Behavior. The International Journal for the Psychology of Religion 21, 85-96 (2011).

[32] Henrich, J. et al. Markets, religion, community size, and the evolution of fairness and punishment. Science 327, 1480-1484 (2010).

[33] Chen, D. L. Club Goods and Group Identity: Evidence from Islamic Resurgence during the Indonesian Financial Crisis. Journal of Political Economy 118, 300354 (2010).

[34] Sosis, R. \& Bulbulia, J. The behavioral ecology of religion: the benefits and costs of one evolutionary approach. Religion 41, 341-362 (2011).

[35] Foley, R. \& Lahr, M. M. Mode 3 technologies and the evolution of modern humans. Cambridge Archaeological Journal 7, 3-36 (1997). 
[36] Hill, K., Barton, M. \& Hurtado, A. M. The emergence of human uniqueness: Characters underlying behavioral modernity. Evolutionary Anthropology: Issues, News, and Reviews 18, 187-200 (2009).

[37] Powell, A., Shennan, S. \& Thomas, M. G. Late Pleistocene Demography and the Appearance of Modern Human Behavior. Science 324, 1298-1301 (2009).

[38] Sterelny, K. From hominins to humans: how sapiens became behaviourally modern. Philosophical Transactions of the Royal Society of London. Series B, Biological sciences 366, 809-822 (2011).

[39] Bulbulia, J. Spreading order: religion, cooperative niche construction, and risky coordination problems. Biology \& Philosophy 27, 1-27 (2012).

[40] Sosis, R. \& Alcorta, C. Signaling, solidarity, and the sacred: The evolution of religious behavior. Evolutionary Anthropology: Issues, News, and Reviews 12, 264-274 (2003).

[41] Mesoudi, A. \& Thornton, A. What is cumulative cultural evolution? Proceedings. Biological sciences 285, 20180712 (2018).

[42] Yeh, D. J., Fogarty, L. \& Kandler, A. Cultural linkage: the influence of package transmission on cultural dynamics. Proceedings. Biological sciences / The Royal Society 286, 20191951 (2019).

[43] Guthrie, S. et al. A cognitive theory of religion [and comments and reply]. Current Anthropology 21, 181-203 (1980).

[44] Cosmides, L. \& Tooby, J. Metarepresentations: A multidisciplinary perspective, chap. Consider the Source: The Evolution of Adaptationsfor Decoupling and Metarepresentation (Oxford University Press, New York, 2000).

[45] Foster, K. R. \& Kokko, H. The evolution of superstitious and superstition-like behaviour. Proceedings of the Royal Society B: Biological Sciences 276, 31-37 (2009). 
[46] Bietti, L. M., Tilston, O. \& Bangerter, A. Storytelling as Adaptive Collective Sensemaking. Topics in Cognitive Science 11, 710-732 (2019).

[47] Smith, D. et al. Cooperation and the evolution of hunter-gatherer storytelling. Nature Communications 8, 1853 (2017).

[48] Watts, J. et al. Broad supernatural punishment but not moralizing high gods precede the evolution of political complexity in austronesia. Proceedings of the Royal Society B: Biological Sciences 282, 20142556 (2015).

[49] Apriasz, R., Krueger, T., Marcjasz, G. \& Sznajd-Weron, K. The Hunt Opinion Model-An Agent Based Approach to Recurring Fashion Cycles. PLoS One 11, e0166323 (2016).

[50] Menger, K. On the Origin of Money. The Economic Journal 2, 239 (1892).

[51] Bauch, C. T. \& Earn, D. Vaccination and the theory of games. Proceedings of the National Academy of Sciences of the United States of America 101, 1339113394 (2004).

[52] Fortunato, L. Reconstructing the history of marriage strategies in IndoEuropean-speaking societies: monogamy and polygyny. Human biology 83, 87-105 (2011).

[53] Frasser, C. \& Guzmán, G. What do we call money? An appraisal of the money or non-money view. Journal of Institutional Economics 16, 25-40 (2020).

[54] Shiller, R. J. Popular economic narratives advancing the longest U.S. expansion 2009-2019. Journal of policy modeling (2020).

[55] Norenzayan, A. \& Shariff, A. F. The origin and evolution of religious prosociality. Science 322, 58-62 (2008).

[56] Traulsen, A. \& Nowak, M. A. Evolution of cooperation by multi-level selection. Proceedings of the National Academy of Sciences USA 103, 10952-10955 (2006). 
[57] Harrison, G. F. et al. Natural selection contributed to immunological differences between hunter-gatherers and agriculturalists. Nature Ecology \& Evolution 3, 1253-1264 (2019).

[58] Armelagos, G. J., Goodman, A. H. \& Jacobs, K. H. The origins of agriculture: Population growth during a period of declining health. Population and Environment 13, 9-22 (1991).

[59] Pearce-Duvet, J. M. C. The origin of human pathogens: evaluating the role of agriculture and domestic animals in the evolution of human disease. Biological Reviews 81, 369-382 (2006).

[60] Wolfe, N. D., Dunavan, C. P. \& Diamond, J. Origins of major human infectious diseases. Nature 447, 279-283 (2007).

[61] Hauert, C., Holmes, M. \& Doebeli, M. Evolutionary games and population dynamics: maintenance of cooperation in public goods games. Proceedings of the Royal Society B 273, 2565-2570 (2006).

[62] Gokhale, C. S. \& Hauert, C. Eco-evolutionary dynamics of social dilemmas. Theoretical Population Biology 111, 28-42 (2016).

[63] Knuth, D. E. Two notes on notation. arXiv.org (1992).

[64] Hofbauer, J. \& Sigmund, K. Evolutionary Games and Population Dynamics (Cambridge University Press, Cambridge, UK, 1998).

[65] Traulsen, A. \& Hauert, C. Stochastic evolutionary game dynamics. In Schuster, H. G. (ed.) Reviews of Nonlinear Dynamics and Complexity, vol. II, 25-61 (Wiley$\mathrm{VCH}$, Weinheim, 2009).

[66] Hauert, C., Traulsen, A., Brandt, H., Nowak, M. A. \& Sigmund, K. Via freedom to coercion: the emergence of costly punishment. Science 316, 1905-1907 (2007). 


\section{Supplementary material}

\section{Evolutionary dynamics in an infinite population}

In total, there are eight different strategies. The strategies can be enumerated by the generic profile $\left(a_{1}^{\star}, a_{2}^{\star}, u^{\star}\right)$ where $a_{i}^{\star}$ is the hunting strategy of the focal individual $(\star)$ when in reality $i$ and $u^{\star}$ is the preferred reality of the focal individual (objective or alternate here-on referred to simply as reality 1 and 2). The individuals in the tribe form a group of size $G$, and they need to decide which reality they choose to believe. Group decision For the group to decide on a choice of reality, 1 or 2 , we use a frequency-dependent process (for other methods of reality resolution, see below). The group thus choose to believe in the reality 1 with probability,

$$
f\left(k, u^{\star}\right)=\frac{k+\delta_{u^{\star}}}{G} .
$$

and in the reality 2 with probability $1-f\left(k, u^{\star}\right)$. The number of individuals, besides the focal, who believe in reality 1 is denoted by $k$. The Kronecker delta $\delta_{u^{\star}}$ returns 1 if the focal individual prefers reality 1 (i.e. if $u^{\star}=1$ ) and 0 otherwise.

Individual action. After the group decides the reality they believe in, each individual in the group chooses the appropriate action. In the reality 1 , the focal individual is a hare hunter if $\delta_{a_{1}^{\star}}$ return 1 and a stag hunter otherwise. A similar reasoning works for reality $2\left(\delta_{a_{2}^{\star}}\right)$.

Values of Hares and Stags. The value of hunting a Hare is denoted by $P_{H}$. The total probability that an individual gets a hare payoff is then denoted by

$$
\Pi_{H}=P_{H} \sum_{k=0}^{G-1}\left(\begin{array}{c}
G-1 \\
k
\end{array}\right) x_{1}^{k}\left(1-x_{1}\right)^{G-1-k}\left(f\left(k, u^{\star}\right) \delta_{a_{1}^{\star}}+\left(1-f\left(k, u^{\star}\right)\right) \delta_{a_{2}^{\star}}\right)
$$

where $x_{1}=x_{H H 1}+x_{H S 1}+x_{S H 1}+x_{S S 1}$ the sum of the frequencies of individuals believe in reality 1 and thus $1-x_{1}=x_{H H 2}+x_{H S 2}+x_{S H 2}+x_{S S 2}$, the reality 2 believers. The value of a stag is given by $P_{S}$. The focal individual is a stag hunter according to the Individual action section. The group composition is a key determinant of the stag payoff since there is a minimum number of stag hunters necessary $(M)$ for 
where the Iverson bracket [63] is used to test the statement $Q=(l+m+n+o=$ $k) \wedge(p+q+r+s=G-1-k)$ with,

$$
[Q(l, m, n, o, p, q, r, s)]= \begin{cases}1, & \text { if } Q \text { is true } \\ 0, & \text { otherwise. }\end{cases}
$$

The function $\chi\left(u_{\star}\right)$ is a step function which (when $\chi\left(u_{\star}=1\right)$ ) ascertains if the focal individual prefers reality 1 and returns the function $\theta(1+n+o+r+s-M)$ (checking if the number of stag hunters meet the required threshold $M$ ). If $u_{\star}=2$ then the focal individual believes in the reality 2 and $\chi\left(u_{\star}\right)$ returns $\theta(1+m+o+q+s-M)$ (again checking if the number of stag hunters meets the required threshold $M$ ). Putting $P_{\text {comp }}$ together with the rest of the probabilities we get the probability of successfully hunting a stag as,

$$
\Pi_{S}=P_{S} \sum_{k=0}^{G-1}\left(\begin{array}{c}
G-1 \\
k
\end{array}\right) P_{c o m p}\left[f\left(k, u^{\star}\right)\left(1-\delta_{a_{1}^{\star}}\right)+\left(1-f\left(k, u^{\star}\right)\right)\left(1-\delta_{a_{2}^{\star}}\right)\right]
$$

The average payoff of an individual with strategy $\left(a_{1}^{\star}, a_{2}^{\star}, u^{\star}\right)$ is then given simply by,

$$
\pi_{\left(a_{1}^{\star}, a_{2}^{\star}, u^{\star}\right)}=\Pi_{H}+\Pi_{S}
$$

The population dynamics can then be represented by the set of replicator equations [64],

$$
\dot{x}_{i}=x_{i}\left(\pi_{i}-\bar{\pi}\right)
$$
for each strategy $i$. There are eight possible strategies and hence the dynamics resides in a seven-dimensional simplex. 


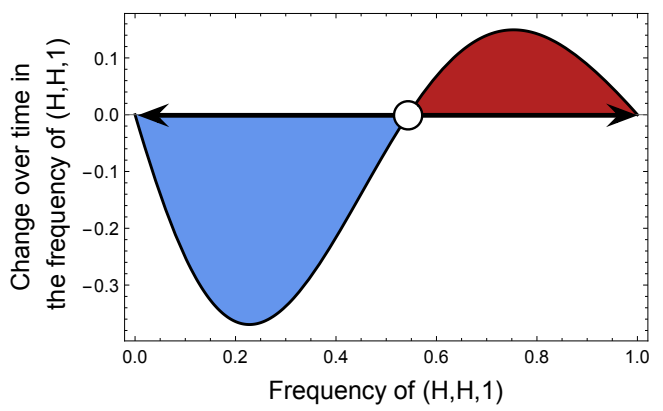

Figure SI.1: Dynamics on the edge between the pure strategies $(H, H, 1)$ and $(S, H, 1)$. If only a small fraction of the population plays the $(\mathrm{H}, \mathrm{H}, 1)$ strategy in a population predominantly composed of $(\mathrm{S}, \mathrm{H}, 1)$ individuals then the group form will consists mostly of stag hunters. While $(\mathrm{S}, \mathrm{H}, 1)$ individuals hunt stag it will be a stable strategy. If the number of $(H, H, 1)$ individuals is above the unstable threshold then stag hunting is not viable since the minimum number of stag hunters required for a successful hunt will not be present but the $(\mathrm{H}, \mathrm{H}, 1)$ individuals thrive. Parameters are: $G=5, M=4, P_{S}=4 ; P_{H}=1$.

Dynamics between pure states The eight vertices of the simplex represent the pure strategies, homogeneous states where all individuals play the same strategy. We study the dynamics between all the pairwise combinations of these pure states. Assume a population which can have only $(H, H, 1)$ and $(S, H, 1)$ individuals. For a group size $G=5$ with a threshold number of stag hunters required for a successful hunt set at $M=4$ a stag provide a payoff of 4 while a hare is worth 1 . Using these values the average payoff of a $(H, H, 1)$ strategist is simply $\pi_{H H 1}=\left(x_{H H 1}+x_{S H 1}\right)^{4}$. The average payoff for a $(S, H, 1)$ player on the other hand is,

$$
\pi_{\mathrm{SH} 1}=4\left(4 x_{\mathrm{HH} 1} x_{\mathrm{SH} 1}^{3}+x_{\mathrm{SH} 1}^{4}\right)
$$
Figure SI.1. In this manner we can describe the dynamics between all the eight vertices, as shown in the main text. 


\section{Evolutionary dynamics in finite populations}

We assume a finite population of size $N$. From this population we choose individuals to form a group of size $G$. If the number of individuals with strategy $j$ is given by $i_{j}$ encapsulated in the vector $\mathbf{i}$, then the average payoff of a strategy $\left(a_{1}^{\star}, a_{2}^{\star}, u^{\star}\right)$ is given by,

$$
\begin{aligned}
\pi_{\left(a_{1}^{\star}, a_{2}^{\star}, u^{\star}\right)=} & \sum_{k=0}^{G-1}\left(P_{H} \frac{\left(\begin{array}{c}
i_{1} i_{3} i_{5} i_{7} \\
k
\end{array}\right)\left(\begin{array}{l}
i_{2} i_{4} i_{6} i_{8} \\
G-1-k
\end{array}\right)}{\left(\begin{array}{l}
N-1 \\
G-1
\end{array}\right)}\left(f\left(k, u^{\star}\right) \delta_{a_{1}^{\star}}+\left(1-f\left(k, u^{\star}\right)\right) \delta_{a_{2}^{\star}}\right)\right. \\
& \left.P_{S} P_{\text {comp }}\left(k, u^{\star}, \mathbf{i}\right)\left(f\left(k, u^{\star}\right)\left(1-\delta_{a_{1}^{\star}}\right)+\left(1-f\left(k, u^{\star}\right)\right)\left(1-\delta_{a_{2}^{\star}}\right)\right)\right) .
\end{aligned}
$$

The composition of the group $P_{\text {comp }}$ is reevaluated for finite populations as,

$$
P_{\text {comp }}=\sum_{\substack{l=0 \\
m=0 \\
n=0 \\
n=0}}^{k} \sum_{\substack{p=0 \\
q=0 \\
r=0}}^{G-1-k} \frac{\left(\begin{array}{c}
i_{1} \\
l
\end{array}\right)\left(\begin{array}{c}
i_{2} \\
p
\end{array}\right)\left(\begin{array}{c}
i_{3} \\
m
\end{array}\right)\left(\begin{array}{c}
i_{4} \\
q
\end{array}\right)\left(\begin{array}{c}
i_{5} \\
n
\end{array}\right)\left(\begin{array}{c}
i_{6} \\
r
\end{array}\right)\left(\begin{array}{c}
i_{7} \\
o
\end{array}\right)\left(\begin{array}{c}
i_{8} \\
s
\end{array}\right)}{\left(\begin{array}{c}
N-1 \\
G-1
\end{array}\right)} \chi\left(u^{\star}\right)[Q(l, m, n, o, p, q, r, s)]
$$

again with $Q$ as defined in Eq. (SI.4). With this approach we can calculate the average payoff of each strategy when playing with another strategy. However for finite populations, we convert the payoff $\pi_{i}$ of a strategy $i$ to its fitness $\psi_{i}$ via a mapping of the form $\psi_{i}=1+\omega \pi_{i}$ where $i$ encompasses the strategies encoded by $\left(a_{1}^{\star}, a_{2}^{\star}, u^{\star}\right)$. Such a combination with $\omega$ allows us to tune the impact of the game on the fitness [65]. If $\omega$ the selection intensity is very low $\omega \rightarrow 0$ then the strategies are neutral with respect to each other. Evolutionary dynamics would then be a random walk between the eight strategies. On the other hand for $\omega \rightarrow 1$ the game completely determines the difference between the strategy fitness. All of this definitely assumes that the strategies do not go extinct, i.e. the mutation probability is non-zero $\mu>0$.

Assuming small mutation rates $\mu \rightarrow 0$, the dynamics typically takes place between two strategies only. Hence a pairwise comparison of the fitnesses of the strategies proves to be instructive. The fitness of a strategy $i$ playing against strategy $j$ is given by $\psi_{i, j}$ This allows us to calculate the fixation probability of a single strategy $i$ player 
in a population of $N-1$ strategy $j$ players as,

$$
\rho_{i, j}=\frac{1}{\sum_{k=1}^{N-1} \prod_{m=1}^{k} \frac{\psi_{j, i}}{\psi_{i, j}}}
$$

Collating the fixation probabilities between all pairwise combinations provides us with the following transition matrix $\mathbf{A}$,

$\mathbf{A}=\left(\begin{array}{cccccccc}1-\sum \text { column } & \rho_{1,2} & \rho_{1,3} & \rho_{1,4} & \rho_{1,5} & \rho_{1,6} & \rho_{1,7} & \rho_{1,8} \\ \rho_{2,1} & 1-\sum \text { column } & \rho_{2,3} & \rho_{2,4} & \rho_{2,5} & \rho_{2,6} & \rho_{2,7} & \rho_{2,8} \\ \rho_{3,1} & \rho_{3,2} & 1-\sum \text { column } & \rho_{3,4} & \rho_{3,5} & \rho_{3,6} & \rho_{3,7} & \rho_{3,8} \\ \rho_{4,1} & \rho_{4,2} & \rho_{4,3} & 1-\sum \text { column } & \rho_{4,5} & \rho_{4,6} & \rho_{4,7} & \rho_{4,8} \\ \rho_{5,1} & \rho_{5,2} & \rho_{5,3} & \rho_{5,4} & 1-\sum \text { column } & \rho_{5,6} & \rho_{5,7} & \rho_{5,8} \\ \rho_{6,1} & \rho_{6,2} & \rho_{6,3} & \rho_{6,4} & \rho_{6,5} & 1-\sum \text { column } & \rho_{6,7} & \rho_{6,8} \\ \rho_{7,1} & \rho_{7,2} & \rho_{7,3} & \rho_{7,4} & \rho_{7,5} & \rho_{7,6} & 1-\sum \text { column } & \rho_{7,8} \\ \rho_{8,1} & \rho_{8,2} & \rho_{8,3} & \rho_{8,4} & \rho_{8,5} & \rho_{8,6} & \rho_{8,7} & 1-\sum \text { column }\end{array}\right)$

The normalised right eigenvector of A corresponding to the largest eigenvalue (which is 1) provides the stationary distribution of the system [66]. This analytical result is plotted as full lines in Figure SI.2 for a given choice of parameters.

\section{Alternative decision making rules}

We have assumed that the hunting party decides the choice of reality, and hence the ensuing actions of the involved individuals via a frequency dependent process (Eq. (SI.1)). In this section describe the dynamics and the eventual distribution of the strategies when the group uses alternative methods for reality consensus forming.

\section{Fixed probability of realising a reality}

Instead if the group instead decides the choice of reality with a fixed probability then the outcome of such a process is qualitatively different than the one discussed in the main text. For a fixed value of 0.5 we show the dynamics for infinitely large populations in Fig. SI.4 
Believe in reality 1

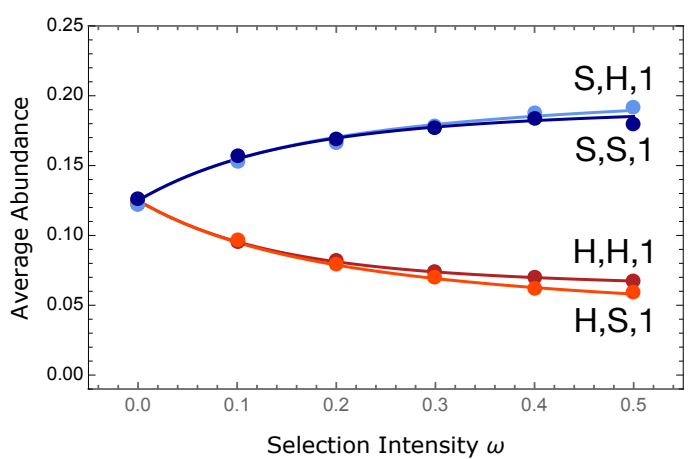

Believe in reality 2

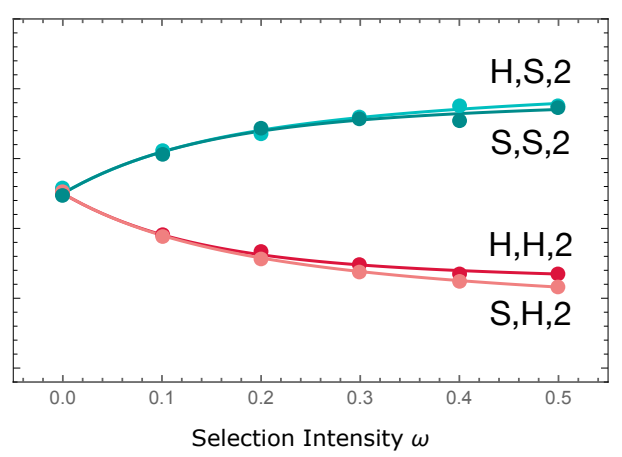

Figure SI.2: Average abundance in the long run. In the long run the strategies in the population stabilise at the proportions which can be calculated analytically (lines) and the results supported by individual based simulations (symbols). For a population of size 16 , and a small mutation probability of $\mu=10^{-3}$, the average abundance of the eight different strategies is denoted above for a variety of selection intensities (after $2 \times 10^{9}$ time-steps). The fitness of each type $i$ is given by $\psi_{i}=1+\omega \pi_{i}$, where $\omega$ is the selection intensity. For $\omega=0$ selection is neutral and all strategies exist in equal proportions $\left(1 / 8^{\text {th }}=0.125\right)$. As selection increases, we see the prevalence of the stag hunters in the population, irrespective of their belief. Parameters are $N=16, G=5$, $M=4, P_{S}=4$ and $P_{H}=1$.

\section{Majority vote}

Whereas the frequency dependent nature of decision making introduces a level of stochasticity in the system, a majority vote removes the ambiguity. If the number of objective reality, 1 , believers form a majority, i.e. $k+\delta_{u^{\star}}>G / 2$ then we assume that the group decides on the inter-subjective reality to be reality 1 (and 2 otherwise).

Majority vote is denoted by a modification of the group decision function to:

$$
f\left(k, u^{\text {star }}\right)= \begin{cases}1, & \text { if } k+\delta_{u^{\star}}>G / 2 \\ 0, & \text { otherwise. }\end{cases}
$$

For a given parameter set the resulting deterministic dynamics between the pure states are shown in Fig. SI.5. Interestingly we see a feedback to the all hare states 


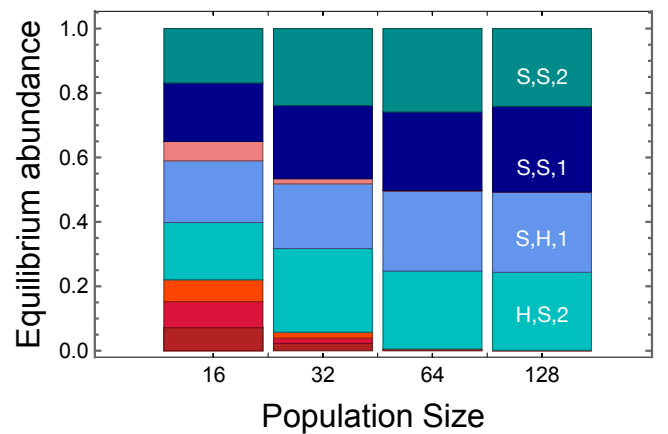

Figure SI.3: Equilibrium abundance across population sizes. For increasing population size, the effect of drift gets diluted and the deterministic equilibrium of the system emerges, which is composed of individuals hunting stags. Simulation parameters besides the changing population size are $\omega=0.4, G=5, M=4, P_{S}=4$ and $P_{H}=1$ with $\mu=10^{-3}$. The system status is reported after $5 \times 10^{6}$ time-steps.

from the jump states of $(\mathrm{H}, \mathrm{S}, 1)$ and $(\mathrm{S}, \mathrm{H}, 2)$. In the long run however, the population

will escape this cycling and end up in the stag hunting state as seen in Fig. SI.6.

\section{Costly beliefs}

If the secondary belief, the subjective reality is cognitively costly, the individual who prefer 2 would pay a cognitive cost. As the cost increases indeed the alternative belief will be harder to fix in the population. However the alternative belief still acts as a catalyst Fig. SI.7. It appears in a finite population by chance but spreads as it is still better to hunt stags than hares. However when everyone is hunting stags, the cognitive cost of maintaining the belief reduces the frequency of the believers. For increasing costs clearly the belief declines however the population is left transformed in a stag equilibrium. 


\section{Group reality chosen with a fixed probability 0.5}

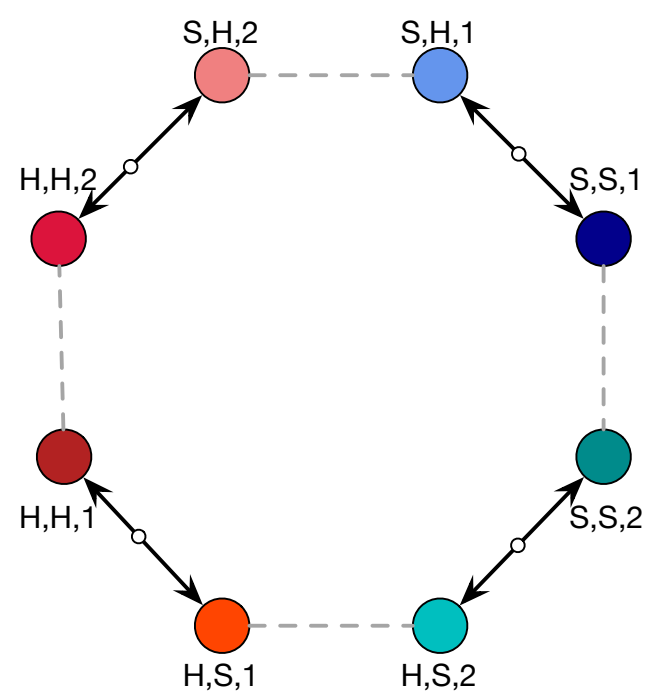

Figure SI.4: Deterministic dynamics for a fixed group decision. Instead of a frequency-dependent process as in Eq. (SI.1), if the reality is decided as 1 with probability 0.5 then the eventual outcome is as shown in the figure. We do not show the links in the interior as all of them consist of an unstable equilibrium and are hence impassable. A population starting at $(H, H, 1)$ will be stuck in the hare equilibrium $(H, H, \star)$. The appearance of the unstable fixed points along the edges lock the population in pure hare or pure stag states. Parameters used here are, $G=5, M=4, P_{S}=4$ and $P_{H}=1$ with the function $f\left(k, u^{\star}\right)=0.5$. Qualitatively similar dynamics are obtained for other fixed values of $f\left(k, u^{\star}\right)$. 
Group reality chosen with a majority vote

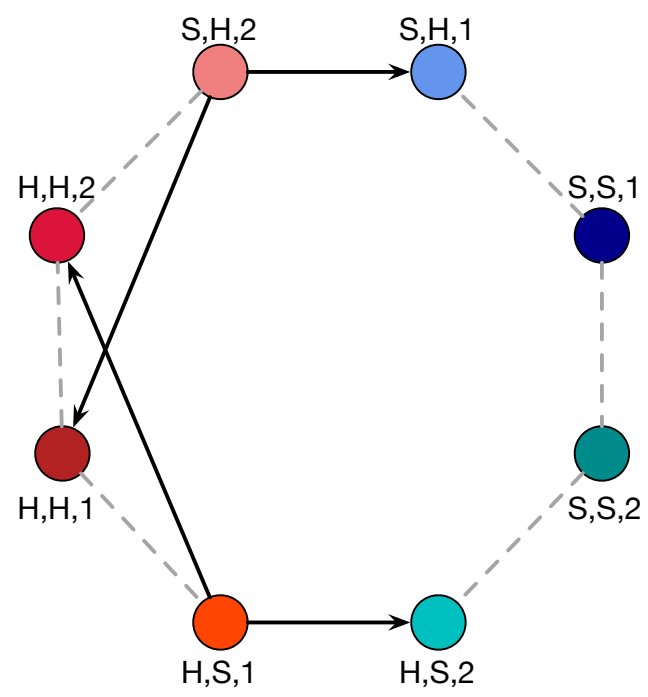

Figure SI.5: Deterministic dynamics for a majority vote decision. Instead of a frequency-dependent process as in Eq. (SI.1), the group reality is chosen by a majority vote. The group reality is then the one which more than $G / 2$ members prefer. In this case we see the appearance of a loop where $(\mathrm{S}, \mathrm{H}, 2)$ and $(\mathrm{S}, \mathrm{H}, 1)$ can lead back to the all hare hunters. However, in the long run, stag hunting in the ultimate sink for the dynamics. Parameters used here are, $G=5, M=4, P_{S}=4$ and $P_{H}=1$ with the function $f\left(k, u^{\text {star }}\right)$ returns the choses reality if the majority believes in it. 


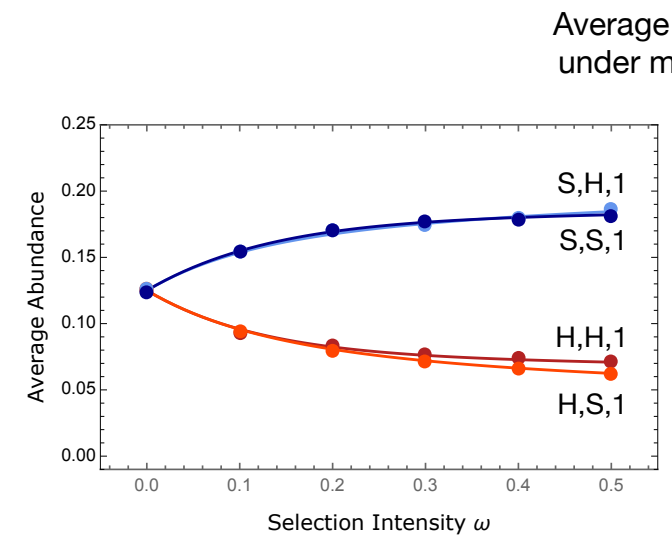

Average abundance under majority vote

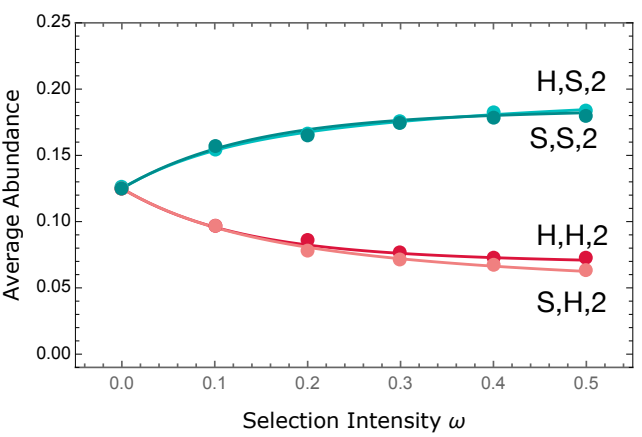

Figure SI.6: Average abundance in the long run under voting. In the long run the strategies in the population stabilise at the proportions which can be calculated analytically (lines) and the results supported by individual based simulations (symbols). For a population of size 16 , and a small mutation probability of $\mu=10^{-3}$, the average abundance of the eight different strategies is denoted above for a variety of selection intensities (after $2 \times 10^{9}$ time-steps). The fitness of each type $i$ is given by $\psi_{i}=1+\omega \pi_{i}$, where $\omega$ is the selection intensity. For $\omega=0$ selection is neutral and all strategies exist in equal proportions $\left(1 / 8^{\text {th }}=0.125\right)$. As selection increases, we see the prevalence of the stag hunters in the population, irrespective of their belief. Parameters are $N=16$, $G=5, M=4, P_{S}=4$ and $P_{H}=1$. 


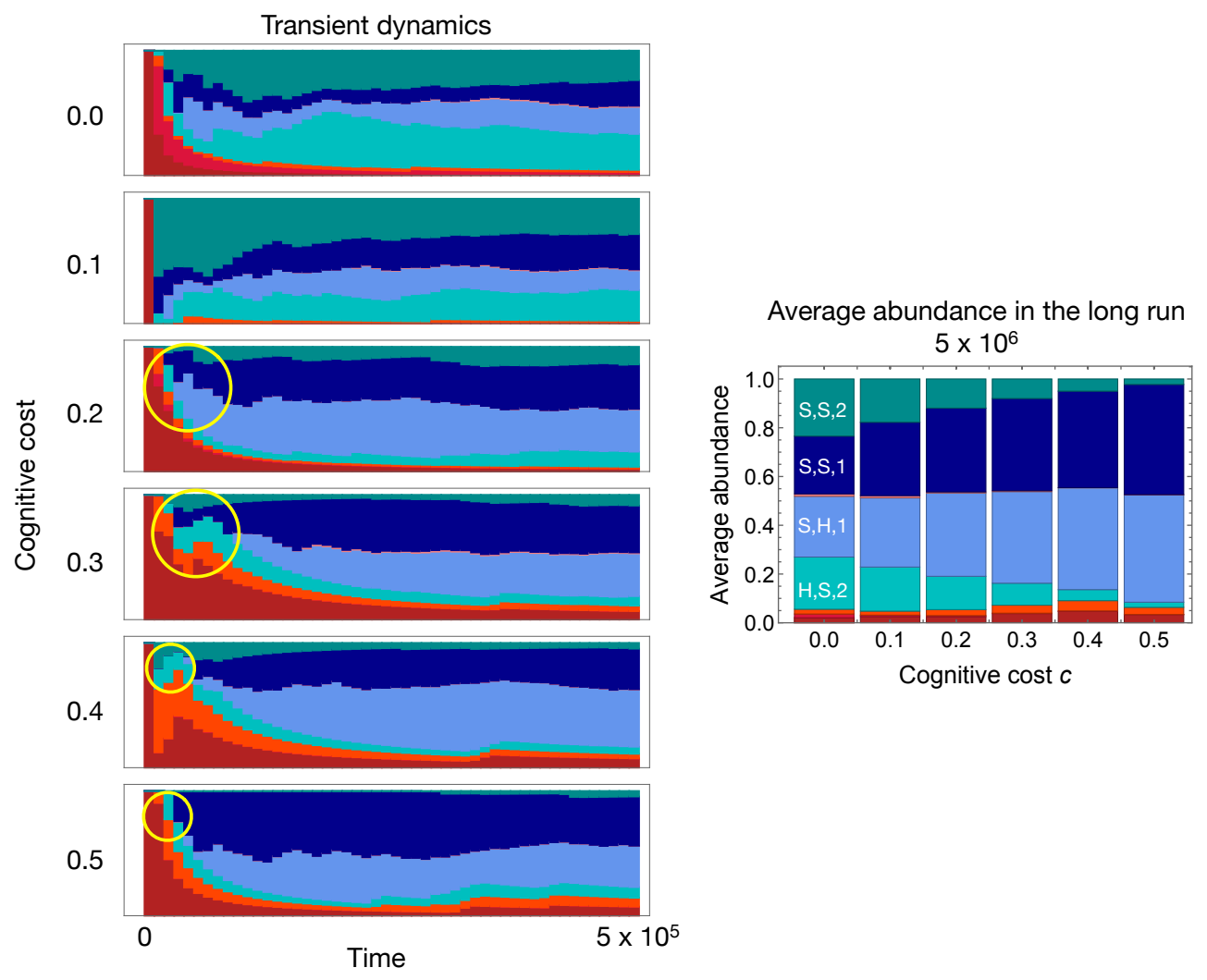

Figure SI.7: Costly beliefs. Even if the preference for the alternative belief accrues a cognitive cost, we show that it helps transform the population into a social group where everyone prefers to hunt stags. The alternative belief acts as a stepping stone (highlighted in the transients on the left by the circles), where the belief in 2 enables the spread of stag hunter who believe in 1 . Thus acting as a true catalyst, the belief helps transform the population and then disappears. The dynamics of the eight strategies for different levels of cognitive costs is shown for a finite population of size 32 , and a small mutation probability of $\mu=10^{-3}$. The equilibrium average abundance of the eight different strategies, which can be calculated analytically is is shown in the right panel for a variety of cognitive costs (after $5 \times 10^{6}$ time-steps). As selection increases, we see the prevalence of the stag hunters in the population, irrespective of their belief. Parameters are $N=16, G=5, M=4, P_{S}=4$ and $P_{H}=1$. The selection intensity is set to $\omega=0.5$. 